\title{
A Rare Cause of Diplopia: Idiopathic Orbital Myositis; A Case Report and Review of the Literature
}

\author{
(D) Şule Gökçe1, Erdem Şimşek2, Sanem Keskin Yılmaz33, Sema Aydoğdu4
}

${ }^{1}$ Ege University Faculty of Medicine, Department of Pediatrics, General Pediatrics Unit, İzmir, Turkey

2Ege University Faculty of Medicine, Department of Pediatrics, Division of Pediatric Neurology, Izmir, Turkey

\begin{abstract}
Orbital myositis is an inflammatory disease affecting the extraocular muscles, especially the medial rectus. The cases are usually referred to clinics with complaints such as diplopia, orbital / periorbital pain, limitation in ocular movements, increased pain with eye movements, proptosis, swelling of the eyelid, hyperemia in the conjunctiva. Orbital myositis is usually idiopathic and autoimmunity is often accused in etiology. It is reported that the disease is associated with polymyositis, thyroid diseases, juvenile rheumatoid arthritis, kawasaki disease, scleroderma, systemic lupus erythematosus, crohn's disease, ankylosing spondylitis. In this article we present a 15-year-old girl who presented with diplopia, pain in both eyes, anomalous head posture, periorbital edema and was diagnosed with idiopathic orbital myositis through history, clinical findings and imaging methods. In this article, it is aimed to draw attention to the diagnosis of idiopathic orbital myositis which is rare in childhood.

Keywords: Orbital myositis; diplopia; anomalous head posture
\end{abstract}

\section{Introduction}

Idiopathic orbital myositis is a syndrome of the acute onset of single or multiple inflammation of the extraocular muscles which can extend to associate with other soft tissues within the orbit. This type of myositis is represented in a broad clinical classification of idiopathic orbital inflammatory pseudotumor [1]. However, it should bekept in mind that idiopathic orbital inflammation and nonspecific orbital inflammation overlap considerably, and occasionally the two entities can be used to be interchangeably. Idiopathic orbital myositis, is a subgroup of idiopathic orbital inflammatory pseudotumor, might be described as a clinical syndrome of inflammation where when the extraocular muscles are the major site in the orbital area
[2]. In addition to muscle tissue, the disease can also affect other structures of the orbit including fat, lacrimal glands, connective tissues [3]. The etiology is not clear yet. However, it is reported to be associated with polymyositis, thyroid diseases, juvenile rheumatoid arthritis, kawasaki disease, scleroderma, SLE, Crohn's disease, ankylosing spondylitis. It has also been shown that there has been an association with several diseases [4,5]. Idiopathic orbital myositis accounts for $6-17 \%$ of cases in childhood, which is the third most common cause of unilateral proptosis after thyroid gland associated eye disease and lymphoproliferative disease [6,7]. It is seen in females two-four times more than males and causes inflammation in one or more extraocular muscles. As a result of the inflammation, orbital signs and symptoms, 
such as pain, proptosis, ptosis, periorbital pain, diplopia, ophthalmoplegia, conjunctival hyperemia, ocular injection, periorbital edema, chemosis, and less commonly vision loss can be seen [8]. Diplopia is due to the inadequate contraction of the affected eye muscles that could bring about an anomalous head posture characterized by head tilting to the side opposite the inflamed muscles to prevent diplopia [9]. Diagnosis of idiopathic orbital myositis can be done after ruling out many diseases that might present with orbital inflammation features. In the magnetic resonance imaging (MRI) of idiopathic orbital myositis cases, there are various patterns of extraocular muscle involvement including single or multiple, unilateral or bilateral, localized or diffuse, and involving tendon sparing or not [3]. In this article, a 15-yearold girl with idiopathic orbital myositis that had no form of chronic systemic illness, presented with swelling-redness in the left eye lid, diplopia for fifteen days, and anomalous head posture for one week, has been discussed, and a brief review of the literature has been given.

\section{Case Report}

\section{Case Description}

A previously healthy 15 -year-old girl presented to our pediatric clinic with a 2-week history of swelling-redness, gradually progressing ptosis in the left eye lid, diplopia, and anomalous head posture. There was no history of neurological disorder or past history of vaccine, drug usage, toxin exposure, immunization, preceding head trauma, fever, chills, or fatigue. Her ophthalmologic examination revealed normal visual acuity, and extraocular movement in all directions was intact but painful, especially in the left eye which was hyperemic, and had alternating exotropia with proptosis. There was a motility restriction of the left eye towards the right side. On her examination of primary view position, she complained of diplopia and to cope with the diplopia she took an mild anomalous head position to the right and downwards, with the jaw to the left. There were no signs of external trauma or insect bites to the left eye or surrounding structures (Figures 1 and 2). Direct and indirect light reflexes of the patient were normal in both eyes. Frontal and posterior segment examinations of the eyes and the remainder of the physical examination were unremarkable. Cranial nerve examination produced normal results. The tone and power of the muscles were normal. Deep tendon reflexes were intact, with no signs of meningeal irritation and babinski. Hemoglobin, biochemical investigations, C- reactive protein and sedimentation rate were normal. Autoimmune tests such as antinuclear antibody, antideoxyribonucleic acid, rheumatoid factor, c-anti-neutrophil cytoplasmic antibody were negative. There was no positivity on serological investigations of lyme, brucella, toxoplasma, toxocara and other viral agents. Gadolinium-enhanced

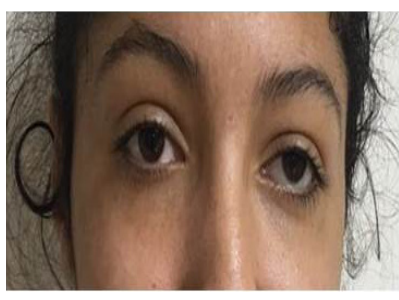

Figure 1. On her primary view position of the left eye which was hyperemic, and had alternating exotropia with proptosis

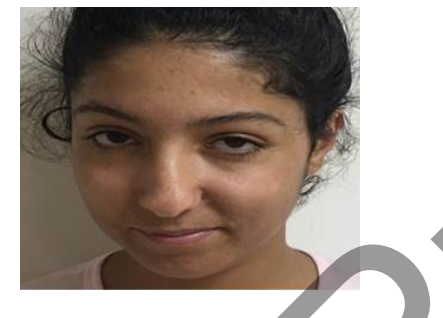

Figure 2. She had mild anomalous head position to the right and downwards, with the jaw to the left There were no signs of external trauma or insect bites to the left eye or surrounding structures

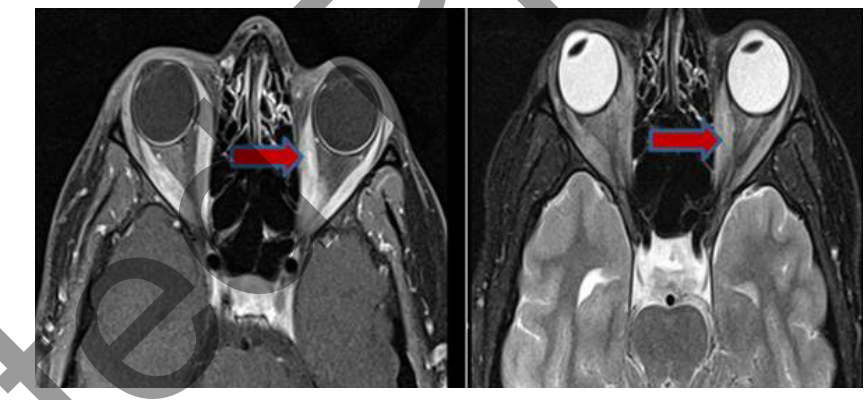

Figures 3 and 4. Magnetic resonance imaging of the orbit showed inflammation in the bilateral extraocular muscles which was more pronounced in the left medial rectus

$\mathrm{T} 1$ and $\mathrm{T} 2$-weighted magnetic resonance imaging of the orbit revealed inflammation in the bilateral extraocular muscles which was more pronounced in the left medial rectus suggesting orbital myositis (Figures 3 and 4). Cranial magnetic resonance imaging was reported as normal. Thyroid ophthalmopathy, intracranial pathologies, rheumatologic diseases and infections were ruled out and the case was diagnosed with idiopathic orbital myositis. Oral $60 \mathrm{mg} /$ day methyl prednisolone therapy was started. On the third day of the treatment, eye edema and proptosis were decreased, and the patient was discharged on hospital day 12 with steroids to be tapered as an outpatient. Methylprednisolone therapy was stopped by gradually reducing within four weeks and policlinic follow-up was performed due to the possibility of recurrence.

\section{Discussion}

We have described a case of a previously healthy child who developed idiopathic orbital myositis associated with no systemic illness. Idiopathic orbital myositis, also called myositis subtype of idiopathic orbital inflammatory pseudotumor, first described by Gleason in 1903 is a myositis resulting in the inflammation of the extraocular muscles without any local or systemic reason [10]. The disease is 
represented in a broad clinical classification of idiopathic orbital inflammation termed by Birch-Hirchfield in 1905 as an orbital pseudotumor condition. The most frequent clinical features of idiopathic orbital myositis are acute or subacute exacerbated orbital pain during eye movements. Others are diplopia, ocular motility restriction, strabismus, eyelid swelling, blepharoptosis, conjunctival injection at the site of tendon insertion, proptosis, periorbital edema, and chemosis. In childhood, it is also reported to be associated with nonspecific signs including headache, emesis, anorexia, lethargy, abdominal pain, and weight loss [3]. It is widely seen in the female gender of 18-40 years of age. In studies, it has been reported that coexistence with diseases such as chronic lymphadenitis, rheumatoid arthritis, systemic lupus erythematosus, polymyositis, thyroid disease, kawasaki disease, scleroderma, crohn's disease, ankylosing spondylitis, ulcerative colitis, celiac disease, and churg-strauss syndrome is possible $[1,3,11]$.

Idiopathic orbital myositis may be acute, chronic or in recurrent form. Unilateral single muscle or multiple bilateral muscles may be involved at the same time. Depending on the location and severity of the disease, the patients might present with symptoms such as pain in the eyes, strabismus, diplopia, propitosis, conjunctival hyperemia, and swellingpain in the eyelids. Additionally, patients may suffer from abnormal head position as a result of diplopia as seen in our case. In a study conducted by Jianhua et al. [3], 86.4\% of the cases with idiopathic orbital myositis were most frequently referred ophthalmologists with oculomotor disorder and strabismus. They have reported that propitosis (68.2\%), congestion-edema (65.9\%) in the conjunctiva, swelling in the eyelids, pain in the eyes, and increased sensation of pressure can also be seen in idiopathic orbital myositis. In the same study, diplopia was found in $43.2 \%$ of the patients.

Diagnosis of idiopathic orbital myositis is based on the history and clinical findings with the detection of one or more extraocular muscular involvement in orbital computerized tomography or orbital magnetic resonance imaging. Diagnosis should be confirmed after excluding thyroid disorders, other orbital inflammatory conditions, vasculitis, sarcoidosis, orbital cellulitis and orbital tumors. Some studies indicated that the medial rectus muscular involvement is the most common involvement, followed by the lateral rectus, superior rectus and inferior rectus muscles, respectively [9].

\section{Conclusion}

Although orbital myositis is a rare disease in childhood, it should be kept in mind in patients presenting with complaints of diplopia, propitosis, periorbital edema and anomalous head posture in differential diagnosis.

\section{Ethics}

\section{Authorship Contributions}

Concept: Ş.G., Design: S.A., Data Collection or Processing: E.Ş., S.Y., Literature Search: S.A., Writing: Ş.G., S.A.

Conflict of Interest: No conflict of interest was declared by the authors.

Financial Disclosure: The authors declared that this study received no financial support.

\section{References}

1. Costa RM, Dumitrascu OM, Gordon LK. Orbital myositis: diagnosis and management. Curr Allergy Asthma Rep 2009; 9: 316-323.

2. Howard Brent, Richard Sidlow. Orbital Myositis: A Case Report and review of the Literature. Clinical Pediatrics 2017; 56: 385388.

Jianhua Yan, Peipei Wu. Idiopathic Orbital Myositis. I Craniofac Surg 2014; 25: 884-887.

4. Kubota T. Orbital myositis. In: Gran IT (eds). Idiopathic Inflammatory Myopathies-Recent Developments. Rijeka,Croatia: InTech, 2011; 123-144.

5. Jordan Spindle, Sunny X. Tang, Brett Davies, et al. Pediatric Idiopathic Orbital Inflammation: Clinical Features of 30 Cases. Ophthal Plast Reconstr Surg 2016; 32: 270-274.

6. Belanger C, Zhang KS, Reddy AK, Yen MT, Yen KG. Inflammatory disorders of the orbit in childhood: a case series. Am I Ophthalmol 2010; 150: 460-463.

7. Weber AL, Romo LV, Sabates NR. Pseudotumor of the orbit. Clinical, pathologic, and radiologic evaluation. Radiol Clin North Am 1999; 37: 151-168.

8. Espinoza GM. Orbital inflammatory pseudotumors: etiology, differential diagnosis, and management. Curr Rheumatol Rep 2010; 12: 443-447.

9. Schoser B. Ocular myositis: diagnostic assessment, differential diagnoses, and therapy of a rare muscle disease- five new cases and review. Clinical Ophthalmology 2007; 1: 37-34.

10. Gleason JE. Idiopathic myositis involving the intraocular muscles. Ophthalmol Rec 1903; 12: 471-478.

11. Wallace ZS, Khosroshahi A, Jakobiec FA, et al. IgC4-related systemic disease as a cause of "idiopathic" orbital inflammation, including orbitalmyositis, and trigeminal nerve involvement. Surv Ophthalmol 2012; 57: 26-33. 